Article

\title{
Five-Year Outcome of Aflibercept Monotherapy for Exudative Age-Related Macular Degeneration with Good Baseline Visual Acuity
}

\author{
Wataru Kikushima, Yoichi Sakurada*D, Atsushi Sugiyama, Seigo Yoneyama, Mio Matsubara, Yoshiko Fukuda \\ and Kenji Kashiwagi $(D$
}

check for updates

Citation: Kikushima, W.; Sakurada, Y.; Sugiyama, A.; Yoneyama, S.; Matsubara, M.; Fukuda, Y.; Kashiwagi, K. Five-Year Outcome of Aflibercept Monotherapy for Exudative Age-Related Macular Degeneration with Good Baseline Visual Acuity. J. Clin. Med. 2021, 10, 1098. https://doi.org/10.3390/ jcm10051098

Academic Editor: Laurent Kodjikian

Received: 28 January 2021

Accepted: 3 March 2021

Published: 5 March 2021

Publisher's Note: MDPI stays neutral with regard to jurisdictional claims in published maps and institutional affiliations.

Copyright: (c) 2021 by the authors. Licensee MDPI, Basel, Switzerland. This article is an open access article distributed under the terms and conditions of the Creative Commons Attribution (CC BY) license (https:/ / creativecommons.org/licenses/by/ $4.0 /)$.
Department of Ophthalmology, University of Yamanashi, Chuo Yamanashi 409-3898, Japan; wkikushima@yamanashi.ac.jp (W.K.); asugiyama@yamanashi.ac.jp (A.S.); syoneyama@yamanashi.ac.jp (S.Y.); miom@yamanashi.ac.jp (M.M.); ysugiyama@yamanashi.ac.jp (Y.F.); kenjik@yamanashi.ac.jp (K.K.)

* Correspondence: sakurada@yamanashi.ac.jp; Tel.: +81-55-273-9657
Keywords: aflibercept monotherapy; polypoidal choroidal vasculopathy; neovascular age-related macular degeneration; good baseline visual acuity

\section{Introduction}

Age-related macular degeneration (AMD), one of the leading causes of legal blindness in advanced countries with older-aged populations [1], is a chronic inflammatory disease with a varied etiology [2]. The intravitreal injection of vascular endothelial growth factor (VEGF) inhibitors has greatly changed the treatment of exudative AMD. It was first reported that a monthly administration of ranibizumab improved the best corrected visual acuity (BCVA) in eyes with exudative AMD in the ANCHOR/MARINA [3,4]. However, a subsequent study, PIER, demonstrated that the quarterly administration of ranibizumab after three-monthly loading injections failed to improve BCVA in eyes with exudative AMD [5]. On the other hand, the PRONTO study demonstrated that monthly monitoring and as-needed reinjection after three monthly loading ranibizumab injections is an effective treatment option for improving BCVA [6]. Currently, anti-VEGF therapy has become the first-line treatment for exudative AMD worldwide; however, repeated injections after the loading phase are required for most eyes [7]. Therefore, the management of recurrence is essential for patients with AMD to maintain good vision for their lifetime.

Aflibercept is a fusion protein that has a stronger binding affinity to VEGF-A in comparison with ranibizumab and bevacizumab. The VIEW1/2 study demonstrated that 
bimonthly aflibercept monotherapy after three monthly loading injections is comparable to monthly ranibizumab monotherapy for visual improvement [8]. Many studies have reported favorable outcomes of intravitreal aflibercept injections (IAI) for exudative AMD with various treatment protocols, including fixed interval dosing, as-needed regimen, and treat-and-extend (TAE) regimens [8-11]. However, the study period was $\leq 24$ months in most studies. In large-scale randomized studies, the inclusion baseline best-corrected visual acuity (BCVA) is $\leq 20 / 40[3,4]$; therefore, there have been few reports investigating the long-term visual outcome in patients secondary to exudative AMD with good baseline visual acuity.

In the present study, we investigated the 5-year visual and anatomic outcomes for patients secondary to exudative AMD with good BCVA $\geq 0.6$, who were initially administrated three monthly aflibercept monotherapy followed by as-needed injection.

\section{Methods}

\subsection{Participants}

A retrospective medical chart review was performed in consecutive treatment-naïve eyes secondary to neovascular AMD (nAMD) or polypoidal choroidal vasculopathy (PCV) with baseline BCVA $\geq 0.6$ in the decimal scale, receiving 3 monthly intravitreal aflibercept injections (IAIs) at the University of Yamanashi Hospital between January 2013 and July 2015. The present study was approved by the Institutional Review Board of the University of Yamanashi and was conducted as per the tenets of the Declaration of Helsinki. Written informed consent was obtained from all patients.

At baseline, all patients underwent comprehensive ophthalmic examinations, including the measurement of BCVA using Landolt chart, intraocular pressure, slit-lamp biomicroscopy with +78-diopter (D) lens, color fundus photography, fluorescein angiography (FA), indocyanine green angiography (ICGA) using a confocal laser scanning system (HRA-2; Heidelberg Engineering, Dossenheim, Germany), and spectral domain optical coherence tomography (SD-OCT) examination (Spectralis version 5.4 HRA + OCT).

We included patients secondary to exudative AMD, including PCV and nAMD. Eyes with nAMD show type 1 or type 2 neovascularization on SD-OCT and the absence of polypoidal lesions on ICGA. PCV shows a solitary or cluster of polypoidal lesions with or without branching vascular networks on ICGA [12]. Lesion size was defined as the greatest linear dimension (GLD). The GLD, which was defined as the fundus lesion covering the dye leak, pigment epithelial detachment, subretinal hemorrhage, and choroidal neovascularization, if present, was determined using the FA image. The central retinal thickness (CRT) was defined as the vertical distance between the inner border of the retinal pigment epithelium and the inner limiting membrane at the center of the macula in the SD-OCT image. The subfoveal choroidal thickness (SCT) was measured as the vertical distance between the outer border of the retinal pigment epithelium and the chorioscleral border, using the enhanced depth imaging mode equipped with HRA2 Spectralis ver 5.4.

The inclusion criteria were as follows: (1) eyes with treatment-naïve exudative AMD, including nAMD and PCV; (2) baseline decimal BCVA $\geq 0.6$ in the Landolt chart; (3) receiving 3 monthly IAI $(2.0 \mathrm{mg} / 0.05 \mathrm{~mL})$; and (4) a follow-up period of 60 months after the initial IAI. The exclusion criteria were (1) previous treatment history for exudative AMD, including intravitreal injection of ranibizumab or photodynamic therapy; (2) eyes that had undergone vitrectomy; (3) eyes with retinal angiomatous proliferation; and (4) other macular abnormalities including myopic choroidal neovascularization $(\mathrm{CNV})$, angioid streaks, and other secondary CNV. If both eyes were eligible, the second eye was included in this study.

\subsection{Follow Up}

All patients received IAIs $(2.0 \mathrm{mg} / 0.05 \mathrm{~mL})$ every 3 months, followed by monthly follow-up requiring SD-OCT examination, as well as routine ophthalmic examination. If residual or recurrent exudation was observed on SD-OCT, retreatment with a single 
IAI was performed. All patients underwent monthly follow-up. When the eyes had not experienced lesion reactivation for more than 12 months, the follow-up interval was extended for a maximum of 2 months until recurrent exudation was observed. If massive subfoveal hemorrhage, dense vitreous hemorrhage, or cataract progression was observed, appropriate surgical procedures, including pars plana vitrectomy and cataract surgery, were performed. The patients who did not complete the 5-year follow-up period were excluded from this study.

\subsection{Genotyping}

We genotyped two major single nucleotide polymorphisms associated with AMD, namely rs800292 in the CFH gene, and rs10490924 in the ARMS2 gene. Genomic DNA was purified using a PUREGENE DNA Isolation Kit (Gentra Systems, Minneapolis, MN, USA) from the peripheral blood of the participants collected at the time of baseline FA/ICGA. Genotyping of the two genes was conducted using TaqMan genotyping assays with a 7300/7500 real-time polymerase chain reaction system (Applied Biosystems, Foster City, CA, USA) following the manufacturer's recommendations as previously described [13]. In detail, TaqMan genotyping assays contain sequence-specific primers to amplify the polymorphic sequence of the target genes, and two minor groove binders to stabilize the samples. Purified wet genomic DNA was mixed with a TaqMan genotyping assay and dispensed onto a reaction plate, and the genotyping with a real-time PCR system was performed. The allelic discriminatin plot was collected and analyzed by three researchers (W.K., Y.S., and S.Y.), and recorded on an anonymous basis.

\subsection{Statistical Analysis}

Statistical analyses were performed using the SPSS for Windows (SPSS, Tokyo, Japan). BCVA measured in the decimal scale with a Landolt chart was converted into a logarithm of the minimal angle resolution (log MAR). The differences between continuous and categorical variables were tested using the Mann-Whitney U test, the Kruskal-Wallis test, or the chi-square test. The paired $t$-test was used to determine the significance of the difference between values before and after treatments. Multivariate logistic regression analysis was performed to investigate the baseline risk factors for retreatment due to residual or recurrent exudation. Cox regression survival analysis was conducted to estimate the relative risk for retreatment. $p$-values less than 0.05 were considered statistically significant.

\section{Results}

\subsection{Change in Log MAR BCVA}

A total of 40 eyes of 40 patients were included in the study. The mean age was $71.8 \pm 8.0$, and 31 patients were male (77.5\%). Table 1 shows the baseline characteristics of the patients. There were 13 patients with nAMD (nAMD group) and 27 with PCV (PCV group). There was no significant difference between the two groups except for the baseline BCVAand the risk allele frequency of ARMS2 and CFH. The patients in the PCV group had better baseline BCVA (Mann-Whitney U test) and lower risk allele frequency for ARMS2 A69S (rs10490924) and CFH I62V (rs800292, Chi-square test, Table 1). However, after adjustment for multiple testing by applying the Benjamini Hochberg method, these three variables were also insignificant.

The change in mean BCVA of the patients in each group during the follow-up period is presented in Figure 1. Although the mean BCVA in each group significantly improved at 12 months from the baseline $(p<0.05)$, the significance disappeared at 24 months, and the final mean BCVA was equivalent to that at baseline $(p=0.17$ in the nAMD group and $p=0.15$ in the PCV group). To evaluate the reason for the decrease in BCVA improvement after month 12, we reviewed the SD-OCT images of the patients with a final BCVA deterioration of $0.3 \log$ MAR or worse. The result revealed that there were $7(17.5 \%)$ patients with severe BCVA deterioration due to various macular or other ocular pathologies, including macular atrophy in two eyes, not achieving dry macula in two eyes, subfoveal hemorrhage 
from PCV in one eye, cataract in one eye, and macular edema secondary to central retinal vein occlusion in one eye.

Table 1. Baseline characteristics of the subject.

\begin{tabular}{|c|c|c|c|c|}
\hline & $\begin{array}{l}\text { All Subjects } \\
\quad n=40\end{array}$ & $\begin{array}{c}\text { nAMD } \\
n=13\end{array}$ & $\begin{array}{l}\text { PCV } \\
n=27\end{array}$ & $\begin{array}{c}p \text {-Value } \\
\text { (nAMD vs. PCV) }\end{array}$ \\
\hline Age & $71.8 \pm 8.0$ & $74.0 \pm 9.3$ & $70.8 \pm 7.2$ & 0.38 \\
\hline Gender male & $31(77.5 \%)$ & $11(84.6 \%)$ & $20(74.1 \%)$ & 0.45 \\
\hline Baseline BCVA & $0.12 \pm 0.09$ & $0.17 \pm 0.07$ & $0.10 \pm 0.09$ & 0.02 \\
\hline Baseline CRT & $377 \pm 155$ & $448 \pm 231$ & $343 \pm 88$ & 0.28 \\
\hline Baseline SCT & $226 \pm 98$ & $185 \pm 56$ & $246 \pm 108$ & 0.10 \\
\hline Baseline GLD & $2767 \pm 1385$ & $3049 \pm 1554$ & $2631 \pm 1306$ & 0.41 \\
\hline \multicolumn{5}{|c|}{ ARMS2 A69S (rs10490924) } \\
\hline $\begin{array}{c}\text { GG:TG:TT } \\
\text { (T allele frequency) }\end{array}$ & $\begin{array}{l}12: 10: 18 \\
(57.5 \%)\end{array}$ & $\begin{array}{c}1: 4: 8 \\
(76.9 \%)\end{array}$ & $\begin{array}{l}11: 6: 10 \\
(48.1 \%)\end{array}$ & 0.015 \\
\hline \multicolumn{5}{|c|}{ CFH I62V (rs800292) } \\
\hline $\begin{array}{c}\text { AA:GA:GG } \\
\text { (G allele frequency) }\end{array}$ & $\begin{array}{l}3: 15: 22 \\
(73.8 \%)\end{array}$ & $\begin{array}{c}0: 3: 10 \\
(88.5 \%)\end{array}$ & $\begin{array}{l}3: 12: 12 \\
(66.7 \%)\end{array}$ & 0.038 \\
\hline
\end{tabular}

nAMD: neovascular age-related macular degeneration, PCV: polypoidal choroidal vasculopathy.

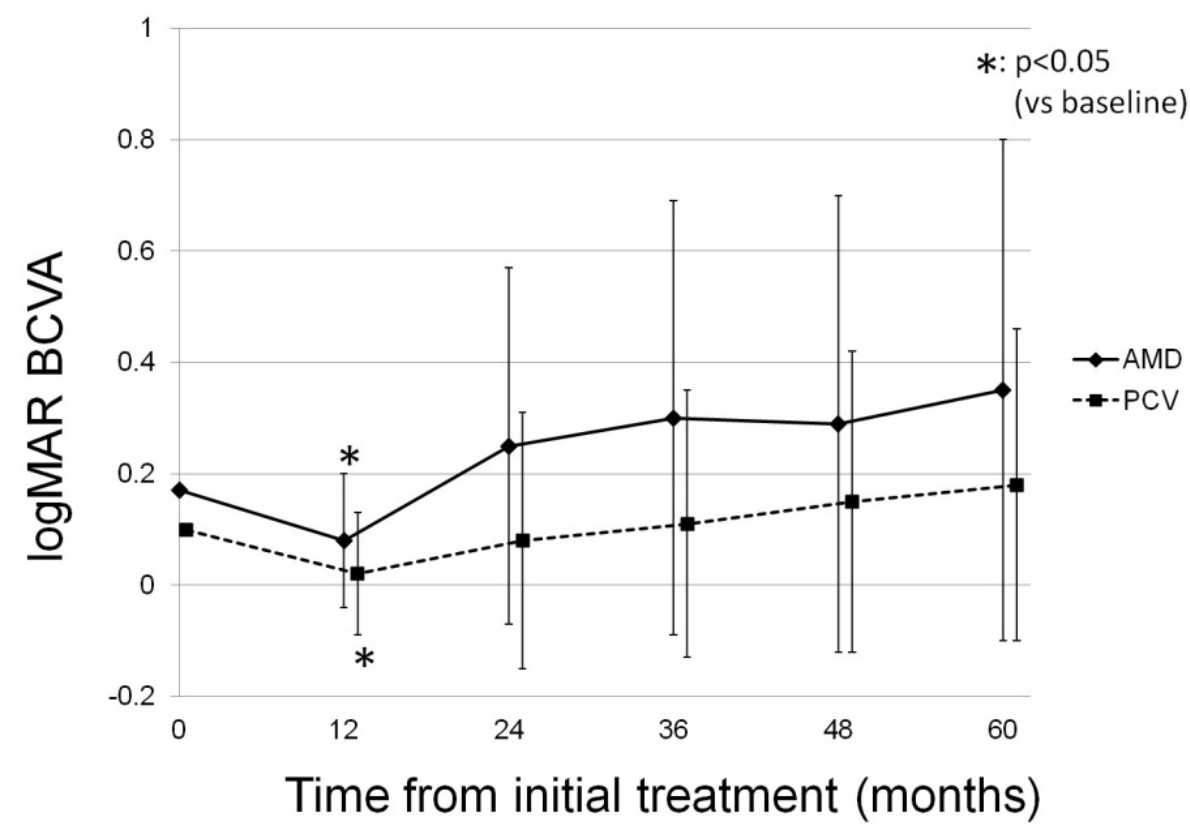

Figure 1. The change in mean best-corrected visual acuity of the participants in each group during the follow-up period. In the neovascular age-related macular degeneration group, the mean BCVA was $0.17 \pm 0.07$ at baseline, and $0.08 \pm 0.12,0.25 \pm 0.32,0.30 \pm 0.39,0.29 \pm 0.41$, and $0.35 \pm 0.45$ at 12 months, 24 months, 36 months, 48 months, and 60 months, respectively ( $p=0.03,0.39,0.25,0.31$, 0.17 , respectively). In the polypoidal choroidal vasculopathy group, the mean BCVA was $0.10 \pm 0.09$ at baseline, and $0.02 \pm 0.11,0.08 \pm 0.23,0.11 \pm 0.24,0.15 \pm 0.27$, and $0.18 \pm 0.28$ at 12 months, 24 months, 36 months, 48 months, and 60 months, respectively $\left(p=1.5 \times 10^{-3}, 0.82,0.80,0.24\right.$, 0.15 , respectively).

Figure 2 presents a representative case with severe vision. The patient required 32 IAIs after initial three monthly injections during a 5-year follow-up period. Though her initial BCVA was 0.8 on the decimal scale, macular atrophy had developed since month 48 , resulting in a deteriorated BCVA of 0.1 at month 60 . 


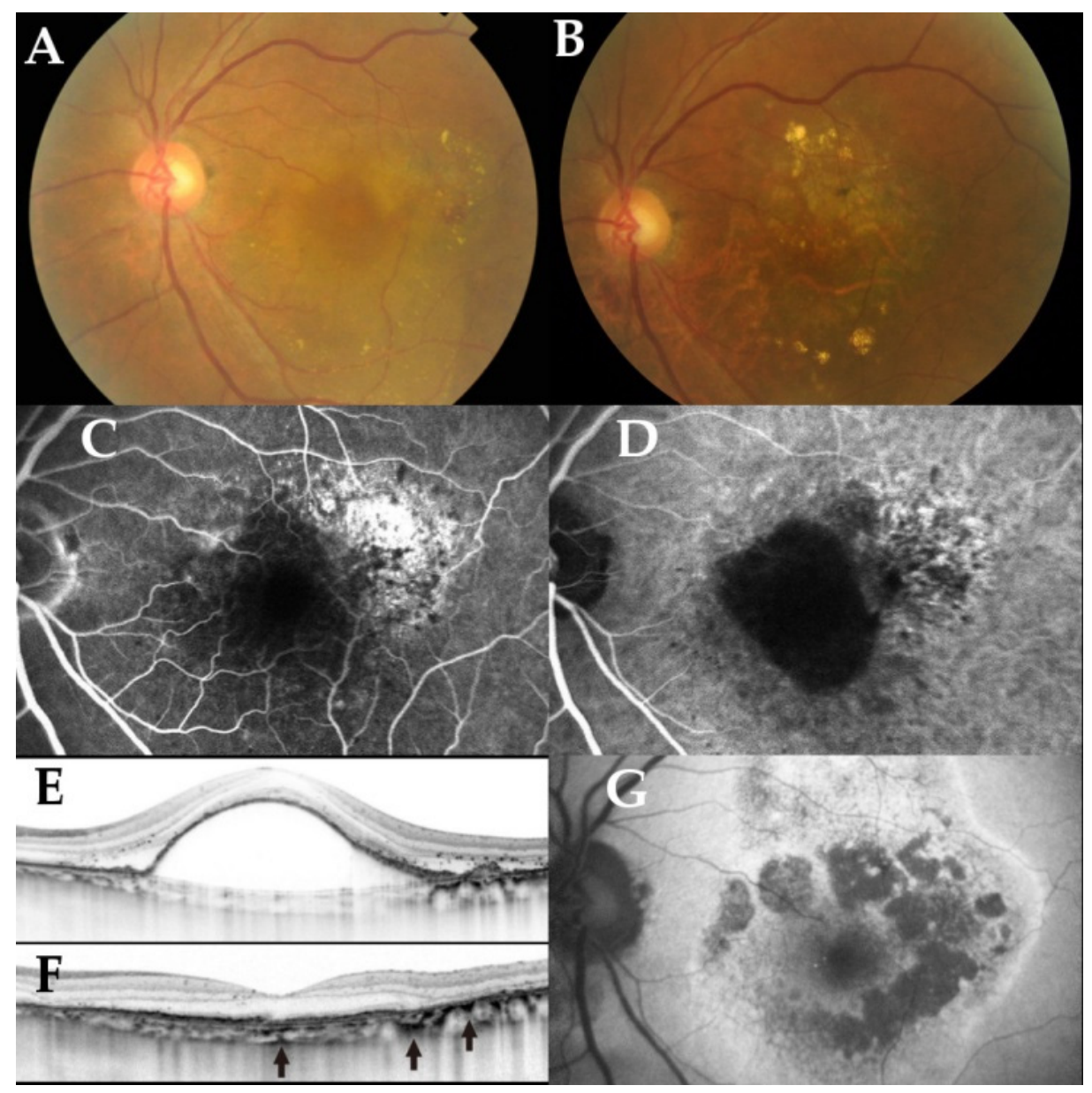

Figure 2. A representative case of a 69-year-old female with type 1 neovascularization in the left eye. (A) Color fundus photography in the left eye at baseline reveals serous retinal detachment (SRD) and pigmental epithelial detachment (PED) in the macula. (B) Color fundus photography in the left eye at 60 months from baseline reveals absorption of SRD and retinal pigment epithelium (RPE) atrophy around the fovea. (C) Fluorescein angiography (FA) of the left eye at baseline demonstrates window defect at the temporal of the fovea. (D) Indocyanine green angiography demonstrates PED including fovea as a hypofluorescent lesion. (E) Spectral domain optical coherence tomography (SD-OCT) of fovea in the left eye at baseline reveals large PED with double-layer sign and SRD. (F) SD-OCT of fovea in the left eye at 60 months from baseline reveals complete resolution of PED and SRD; however, choroidal hypertransmission due to subfoveal RPE atrophy is apparent (black arrows). (G) Fundus autofluorescense of the left eye at 60 months from baseline reveals RPE atrophy as hypoautofluorescent areas around the fovea.

To investigate the factors associated with BCVA change at 60 months, we conducted a multivariate regression analysis as a multiple comparison (Table 2). The results showed that female sex was associated with good BCVA improvement at 60 months. During the follow-up period, four patients underwent cataract surgery. However, the history of cataract surgery was not associated with BCVA improvement at 60 months (Table 2). None of the eyes required vitrectomy.

\subsection{Change in OCT Parameters}

During the follow-up period, the mean CRT of all participants significantly decreased from $376.7 \pm 155.1 \mu \mathrm{m}$ at baseline to $237.1 \pm 62.7 \mu \mathrm{m}$ at 60 months $(p<0.05)$. A significant reduction in CRT was also observed in both the nAMD and PCV groups $(p<0.05)$. Figure 3 illustrates the change in mean CRT in each group. 
Table 2. Multivariate linear regression analysis of factors associated with mean BCVA change at 60 months.

\begin{tabular}{ccc}
\hline Variables & $\beta$-Coefficient & $p$-Value \\
\hline Age & 0.01 & 0.25 \\
Male gender & -0.36 & 0.023 \\
Baseline log MAR BCVA & -0.23 & 0.76 \\
Greatest linear dimension & $-2.8 \times 10^{-6}$ & 0.95 \\
Central retinal thickness & $4.1 \times 10^{-4}$ & 0.41 \\
Subfoveal choroidal thickness & $-4.5 \times 10^{-4}$ & 0.50 \\
Subtype (nAMD = 0, PCV = 1) & 0.02 & 0.91 \\
ARMS2 A69S T allele & $2.0 \times 10^{-3}$ & 0.98 \\
CFH I62V G allele & 0.14 & 0.16 \\
Cataract surgery & 0.046 & 0.82 \\
Total number of IAIs & $5.6 \times 10^{-4}$ & 0.90 \\
\hline
\end{tabular}

ARMS2: age-related maculopathy susceptibility; BCVA: best-corrected visual acuity; $\mathrm{CFH}$ : complement factor $\mathrm{H}$; IAI: intravitreal aflibercept injection; logMAR: logarithm of the minimal angle resolution.

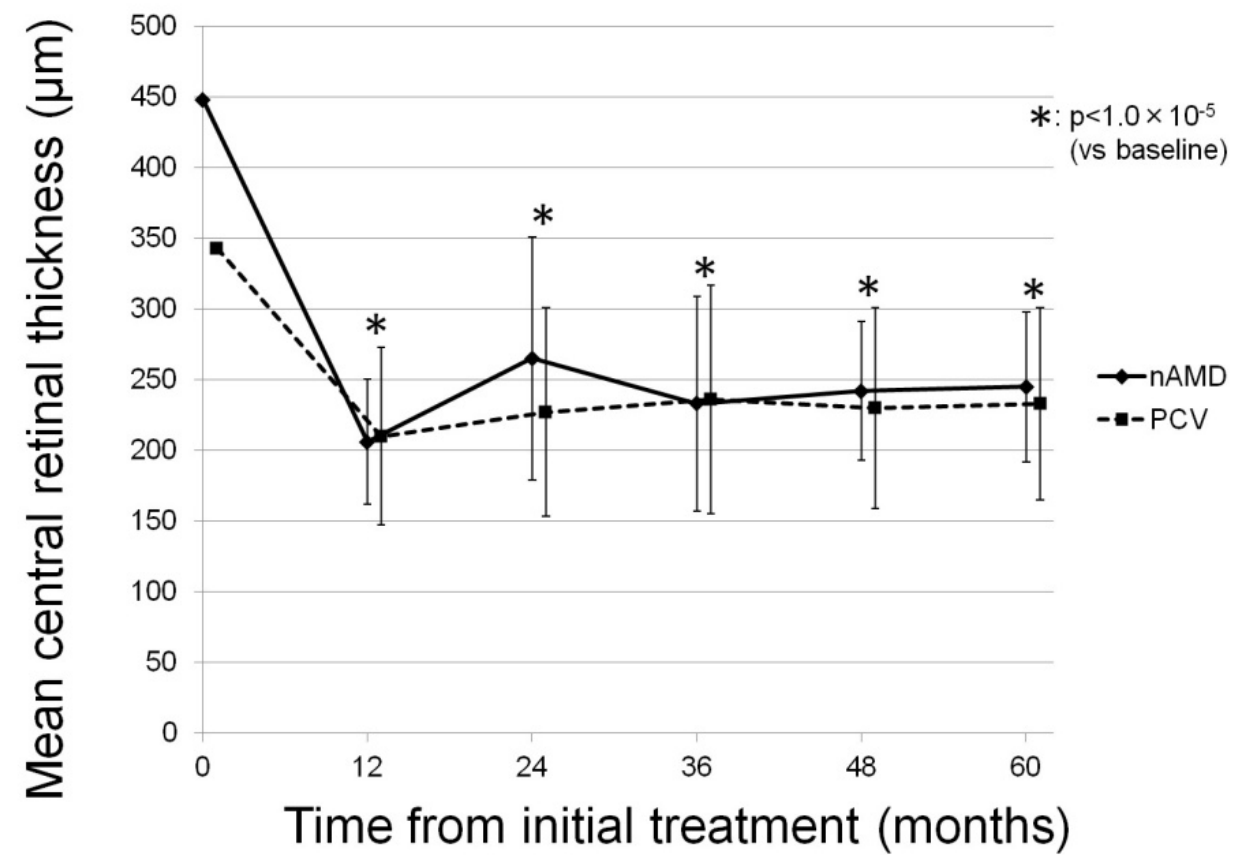

Figure 3. The change in mean central retinal thickness in each group during the follow-up period. Mean central retinal thickness (CRT) shows significant decreases from $448 \pm 231 \mu \mathrm{m}$ at baseline to $206 \pm 44 \mu \mathrm{m}$ at 12 months, $265 \pm 86 \mu \mathrm{m}$ at 24 months, $233 \pm 76 \mu \mathrm{m}$ at 36 months, $242 \pm 49 \mu \mathrm{m}$ at 48 months, and $245 \pm 53 \mu \mathrm{m}$ at 60 months $\left(p=2.5 \times 10^{-3}, 9.1 \times 10^{-3}, 2.4 \times 10^{-3}, 6.9 \times 10^{-3}\right.$, $3.1 \times 10^{-3}$, respectively) in the neovascular age-related macular degeneration group, and mean CRT shows a significant decrease from $343 \pm 88 \mu \mathrm{m}$ at baseline to $210 \pm 63 \mu \mathrm{m}$ at 12 months, $227 \pm 74 \mu \mathrm{m}$ at 24 months, $236 \pm 81 \mu \mathrm{m}$ at 36 months, $230 \pm 71 \mu \mathrm{m}$ at 48 months, and $233 \pm 68 \mu \mathrm{m}$ at 60 months $\left(p=1.0 \times 10^{-5}, 1.0 \times 10^{-5}, 4.0 \times 10^{-4}, 1.0 \times 10^{-5}, 1.0 \times 10^{-5}\right.$, respectively) in the polypoidal choroidal vasculopathy group.

Similar to CRT change, the mean SCT of all participants significantly decreased from $226.0 \pm 97.8 \mu \mathrm{m}$ at baseline to $198.8 \pm 80.0 \mu \mathrm{m}$ at 60 months $(p<0.05)$. In the nAMD group, the mean SCT significantly decreased at 12 months from baseline $(p<0.05)$; however, the final mean SCT reduction was not significant at 60 months ( $p=0.37)$. In contrast, in the PCV group, a significant decrease in mean SCT was maintained throughout the follow-up period $(p<0.05)$. Figure 4 shows the changes in the mean SCT in each group. 


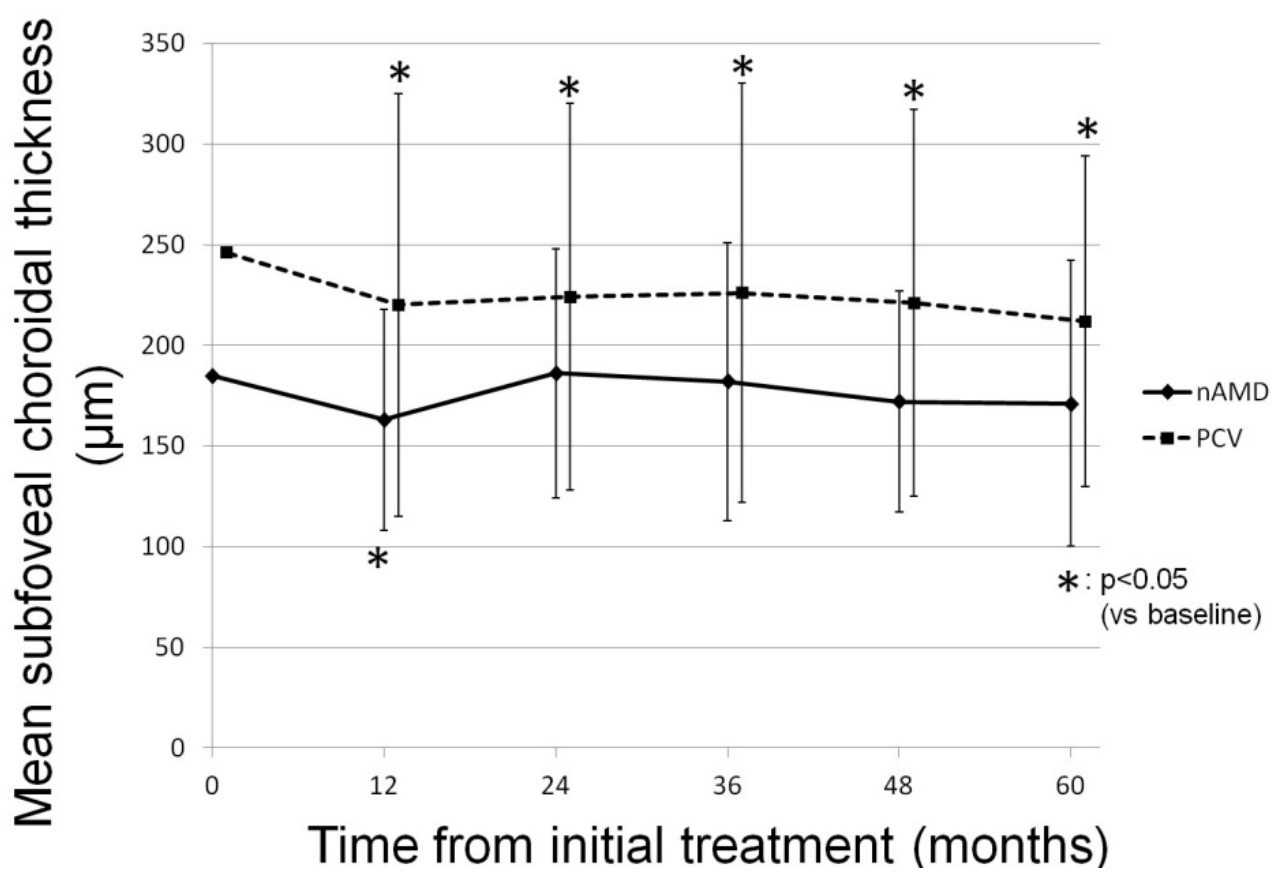

Figure 4. The change in mean subfoveal choroidal thickness in each group during the follow-up period. In the neovascular age-related macular degeneration group, mean subfoveal choroidal thickness (SCT) was $185 \pm 56 \mu \mathrm{m}$ at baseline, and $163 \pm 55 \mu \mathrm{m}, 186 \pm 62 \mu \mathrm{m}, 182 \pm 69 \mu \mathrm{m}, 172 \pm 55 \mu \mathrm{m}$, and $171 \pm 71 \mu \mathrm{m}$ at 12 months, 24 months, 36 months, 48 months, and 60 months, respectively $(p=0.013,0.95,0.82,0.28$, and 0.37 , respectively). In the polypoidal choroidal vasculopathy group, mean SCT showed a significant decrease from $246 \pm 108 \mu \mathrm{m}$ at baseline to $220 \pm 105 \mu \mathrm{m}$ at 12 months, $224 \pm 96 \mu \mathrm{m}$ at 24 months, $226 \pm 104 \mu \mathrm{m}$ at 36 months, $221 \pm 96 \mu \mathrm{m}$ at 48 months, and $212 \pm 82 \mu \mathrm{m}$ at 60 months $\left(p=6.0 \times 10^{-5}, 2.9 \times 10^{-4}, 3.3 \times 10^{-3}, 1.8 \times 10^{-3}, 1.9 \times 10^{-3}\right.$, respectively).

\subsection{Retreatment}

Among the 40 eyes, 37 (92.5\%) needed IAI(s) as retreatment after the loading dose during the 60-month follow-up period. Figure 5 shows the distribution of patients according to the number of total injections required after the loading dose. Table 3 shows the mean number of total injections required after the loading dose and the distribution of mean number of injections year-by-year. There is no significant difference in the mean number of IAIs between the two groups $(21.2 \pm 14.2$ in the neovascular AMD group and $17.1 \pm 13.9$ in the PCV group, $p=0.36$ ). We conducted a multivariate linear regression analysis to investigate the factors associated with the number of total injections required after the loading dose as a multiple comparison. As shown in Table 4, the risk allele of ARMS2 A69S (rs10490924) was the only factor associated with the number of total injections.

To investigate the factors associated with retreatment, a Cox regression survival analysis was conducted. The results showed that the protective allele (G allele) of ARMS2 A69S (rs10490924) was the only factor associated with a retreatment-free period from the initial injection ( $\beta$-coefficient $=0.42, p=0.041$, repeated forward selection method). Other variables including age, sex, baseline BCVA, baseline CRT, baseline SCT, or allele of CFH I62V (rs800292) were not associated with a retreatment-free period. Figure 6 shows the Kaplan-Meier curve for a retreatment-free proportion from the initial injection depending on disease subtypes, ARMS2 A69S, and CFH I62V genotypes. 
14

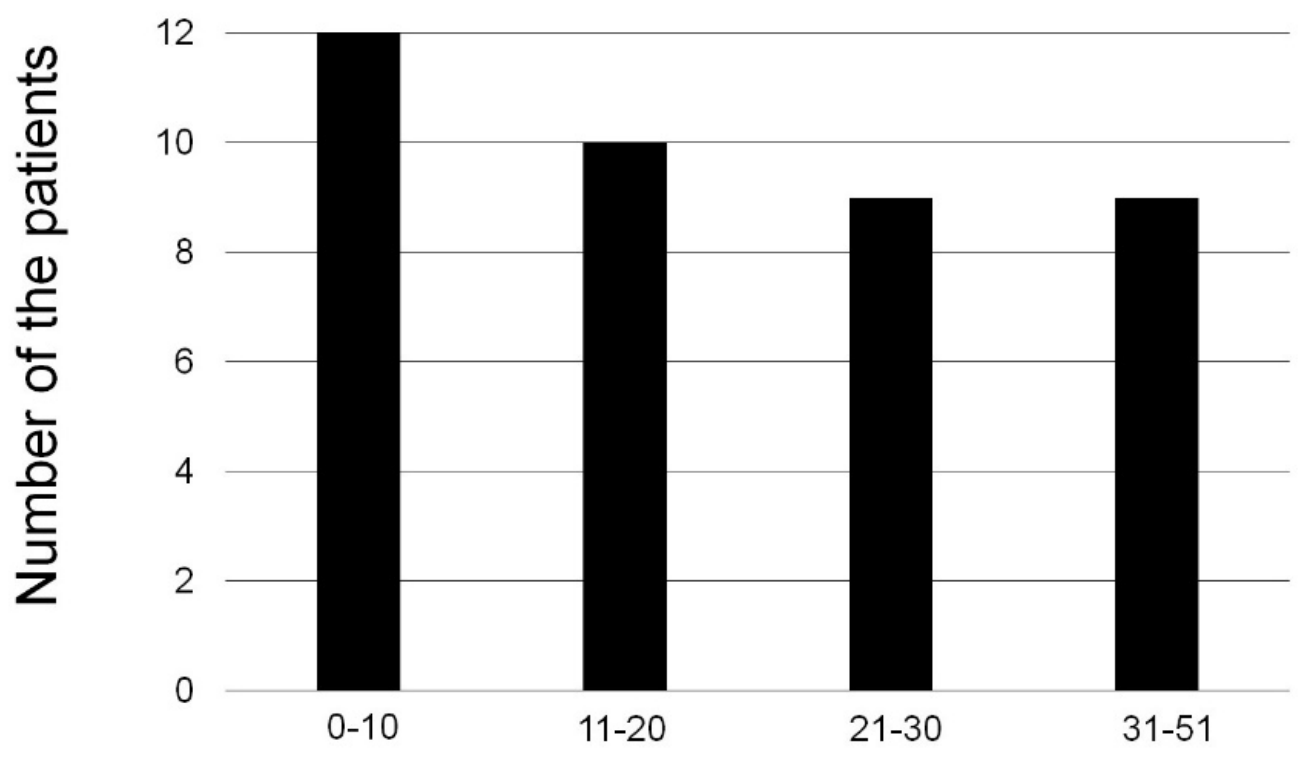

Total number of additional injections

Figure 5. The distribution of patients according to the number of total injections required after the loading dose. In total, 12 (30\%) patients were administered 0-10 injections, 10 (25\%) patients 11-20 injections, 9 (22.5\%) patients 21-30 injections, and 9 (22.5\%) patients were administered 35-51 injections during the follow-up period. The median and mean numbers of intravitreal aflibercept injections required after the loading dose in all patients are 15.0 and $18.4 \pm 14.0$.

Table 3. Mean number of total injections required after the loading dose and the distribution of the mean number of injections year-by-year.

\begin{tabular}{cccccc}
\hline $\begin{array}{c}\text { Total } \\
\mathbf{( 0 - 6 0} \text { Months })\end{array}$ & $\begin{array}{c}\text { 1st Year } \\
\mathbf{( 0 - 1 2} \text { Months) }\end{array}$ & $\begin{array}{c}\text { 2nd Year } \\
\mathbf{( 1 3 - 2 4} \text { Months) }\end{array}$ & $\begin{array}{c}\text { 3rd Year } \\
\mathbf{( 2 5 - 3 6} \text { Months) }\end{array}$ & $\begin{array}{c}\text { 4th Year } \\
\text { (37-48 Months) }\end{array}$ & $\begin{array}{c}\text { 5th Year } \\
\mathbf{( 4 9 - 6 0 ~ M o n t h s ) ~}\end{array}$ \\
\hline $18.4 \pm 14.0$ & $2.4 \pm 2.5$ & $3.8 \pm 3.1$ & $3.7 \pm 3.4$ & $3.8 \pm 3.4$ & $4.4 \pm 3.9$ \\
\hline
\end{tabular}

Table 4. Multivariate linear regression analysis of factors associated with number of total injections.

\begin{tabular}{ccc}
\hline Variables & $\beta$-Coefficient & $p$-Value \\
\hline Age & 0.44 & 0.23 \\
Male gender & -5.61 & 0.36 \\
Baseline log MAR BCVA & 39.3 & 0.18 \\
Greatest linear dimension & $-2.0 \times 10^{-3}$ & 0.32 \\
Central retinal thickness & 0.023 & 0.22 \\
Subfoveal choroidal thickness & -0.029 & 0.27 \\
Subtype (nAMD = 0, PCV = 1) & 7.09 & 0.27 \\
ARMS2 A69S T allele & 6.63 & 0.025 \\
CFH I62V G allele & 0.87 & 0.83 \\
\hline
\end{tabular}

ARMS2: age-related maculopathy susceptibility; BCVA: best-corrected visual acuity; $\mathrm{CFH}$ : complement factor $\mathrm{H}_{;}$ logMAR: logarithm of the minimal angle resolution. 
A
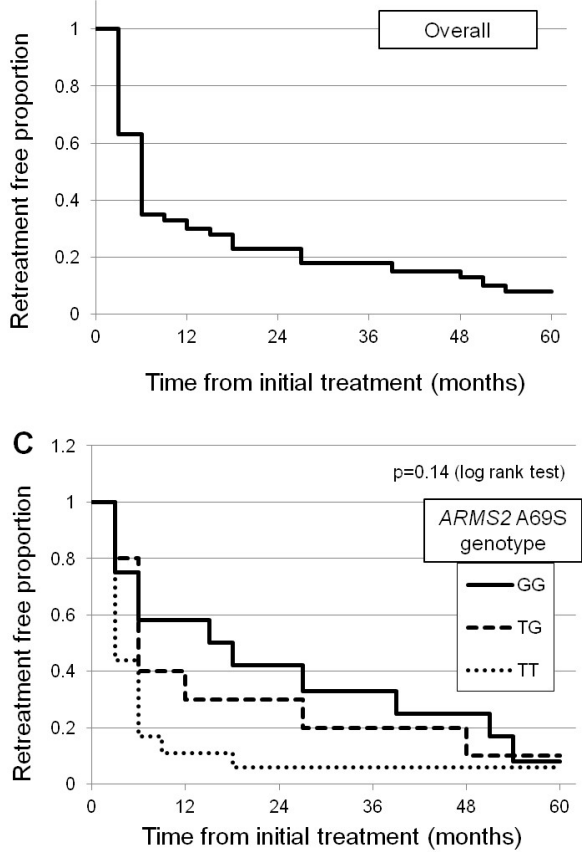

B
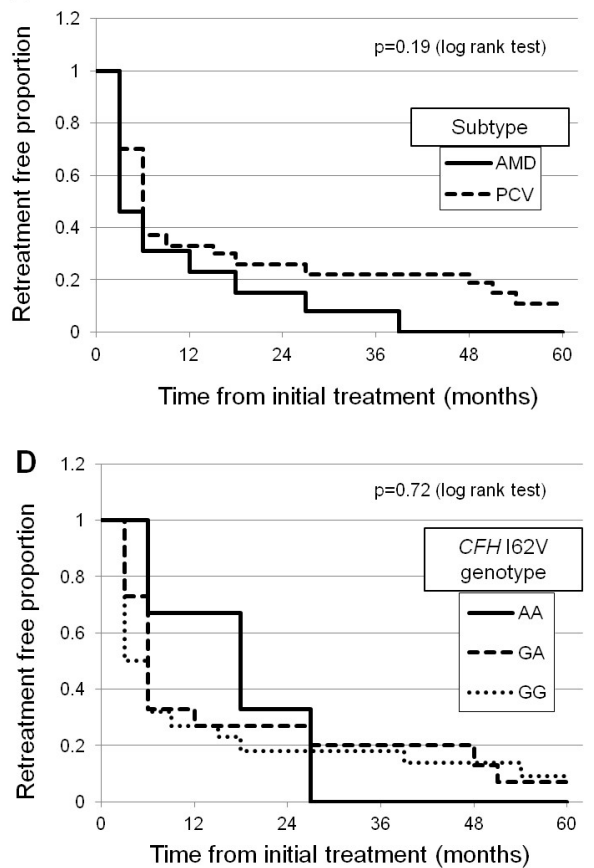

Figure 6. Kaplan-Meier curve regarding retreatment-free proportion from the initial injection. (A) The mean retreatment-free period after the initial intravitreal aflibercept injections (IAI) is $16.1 \pm 18.7$ months, and the retreatment-free proportion at 60 months is $7.5 \%$ in all patients. (B) The mean retreatment-free period is $11.2 \pm 11.5$ months and $18.5 \pm 21.1$ months in the nAMD and PCV groups, respectively. The retreatment-free proportion at 60 months is $0 \%$ and $11 \%$ in the nAMD group and the PCV group, respectively ( $p=0.19$, log-rank test). (C) Mean retreatment-free period is $24.6 \pm 18.8$ months, $18.8 \pm 20.1$ months, and $9.0 \pm 13.2$ months in patients with GG, TG, and TT genotypes of ARMS2 A69S, respectively. The retreatment-free proportion at 60 months is $8 \%, 10 \%$, and $6 \%$ in patients with GG, TG, and TT genotypes of ARMS2 A69S, respectively ( $p=0.14$, log-rank test). (D) The mean retreatment-free period is $17.7 \pm 10.7$ months, $17.4 \pm 19.6$ months, and $15.0 \pm 19.5$ months in patients with AA, AG, and GG genotype of CFH I62V, respectively. The retreatment-free proportion at 60 months is $0 \%, 7 \%$, and $9 \%$ in patients with $\mathrm{AA}, \mathrm{AG}$, and GG genotypes of CFH I62V, respectively ( $p=0.72$, log-rank test).

\section{Discussion}

To the best of our knowledge, this is the first study investigating the long-term treatment outcome of as-needed IAIs for exudative AMD in patients with relatively good initial visual acuity. Our study was a retrospective cohort study; therefore, we consider it as valuable as real-world data.

In the literature, there have been several studies evaluating the long-term efficacy and safety of anti-VEGF agents, including aflibercept, for exudative AMD. Khanani et al. reported on the five-year outcome of an intravitreal anti-VEGF agent treatment using a TAE regimen for nAMD in eyes with baseline vision 20/60 or better. They described that more than $75 \%$ of the patients could maintain their baseline VA, and the mean number of injections during years $1-5$ was $7.9,5.9,5.6,5.9$, and 6.0, respectively [14]. Javidi et al. reported the treatment outcome of a similar intravitreal drug therapy for nAMD using a TAE regimen for up to 7 years [15]. The results were also preferable; the mean BCVA was maintained with a mean number of injections of 7.6 during the first three years, and 5.9 during years 4-7. The difference between our study and these studies is that we selected IAI only for the intravitreal treatment with an as-needed regimen, whereas they had chosen not only aflibercept but also ranibizumab or bevacizumab using the TAE regimen. As a result, the patients in our study achieved similar visual outcomes with relatively fewer additional injections than those in their studies. 
Maguire et al. reported the long-term treatment outcomes of anti-VEGF agents, including bevacizumab and ranibizumab, for treating nAMD in an extended comparison of AMD treatments trial study [16]. They reported that the mean change in visual acuity at 5 years from baseline was -3 letters. In the present study, the mean BCVA changed from $0.12 \pm 0.09$ at baseline to $0.23 \pm 0.35$ at 60 months, and the BCVA change was equivalent to -6 letters on the early treatment diabetic retinopathy study chart. Given that the initial visual acuity in the present study was equal to or better than the decimal BCVA of 0.6 , we consider that the as-needed aflibercept therapy was an effective treatment option for exudative AMD, even in patients with good initial BCVA. However, in the present study, a significant improvement in mean BCVA was apparent at 12 months in both the nAMD and PCV groups; thereafter, the mean BCVA returned to the baseline values and was maintained throughout the follow-up period. We consider that the discrepancy between the insignificant BCVA improvement and the significant reduction in CRT or SCT at month 60 was due to the patients with macular or other pathologies, which were independent of CRT of SCT. In the present study, 7 of 40 patients showed BCVA deterioration of $0.3 \operatorname{logMAR}$ or worse due to these conditions. Even if these patients with severe BCVA deterioration were excluded from the analysis, it did not reach a statistical significance between baseline BCVA and BCVA at 60 months.

During a 60-month follow-up period, 37 (92.5\%) out of 40 eyes needed a minimum of one retreatment. Cox regression analysis revealed that the protective allele of ARMS2 A69S was associated with a retreatment-free period from the initial injection. In a previous study, we investigated the short-term prognostic factors associated with retreatment among patients who received an initial 3-month IAI for exudative AMD [17]. Cox regression analysis revealed that the risk allele of the ARMS2 A69S gene and older age were associated with a shorter retreatment and reactivation time from the initial injection. In the present study, the positive association between the protective allele of ARMS2 A69S and the retreatment-free period from initial injection was also confirmed even in patients with good baseline BCVA. Recently, we reported that the protective allele of ARMS2 A69S was associated with a retreatment-free period in patients with PCV who received the combination therapy involving photodynamic therapy (PDT) and intravitreal injection of anti-VEGF agents $[18,19]$. The differences between the present study and the previous study are the better initial mean BCVA, including the patients with nAMD, and excluding PDT as a treatment modality in the present study. Consequently, there are several differences in the results between the studies. The proportion of the patients who had not required any retreatment was greater in the previous study than in the present study $(27.9 \% \mathrm{vs} .7 .5 \%)$, and the total number of retreatments during a 5-year follow-up period in the previous study was relatively smaller than that in the present study $(7.51 \pm 7.25$ injections and $0.51 \pm 1.01$ combination therapies vs. $18.4 \pm 14.0$ injections). These results might indicate the good potential of PDT in terms of disease stability against PCV. On another front, the preferable results in the present study might shed light on the choice of treatment modality for PCV patients with initial BCVA $\geq 0.8$ in the decimal scale, which had not been included in the previous studies. Moreover, from the previous and present results, it might be inferred that the risk allele of ARMS2 A69S might be associated with lesion reactivation after treatment, regardless of the treatment modality.

There are several limitations to the present study. The first limitation is the retrospective nature of the analysis. The second limitation is the relatively small sample size. The third limitation is the wide fluctuations in the data. The standard deviations of mean BCVA, CRT, and SCT presented in Figures 1-3 were high, and the significant $P$ values presented in the tables were slightly below the threshold of $p<0.05$. These limitations might lead to some uncertainties about the interpretation of the findings. Further prospective analysis of the long-term visual and anatomical outcomes of aflibercept for exudative AMD with a large sample size is necessary. 
In conclusion, IAI is a preferable treatment option for exudative AMD for prolonged disease stabilization, even in patients with good initial visual acuity. The risk allele of the ARMS2 A69S gene is associated with earlier retreatment after the initial three monthly IAIs.

Author Contributions: Conception of the study (Y.S.), Data collection (W.K., Y.F., Y.S., A.S., S.Y., M.M.), Statistical analysis (W.K.). Writing the original draft (W.K.), Review and editing of manuscript (Y.S., K.K.). All authors have read and agreed to the published version of the manuscript.

Funding: This research received no external funding.

Institutional Review Board Statement: This retrospective study was approved by Institutional Review Board in University of Yamanashi.

Informed Consent Statement: Written informed consent was obtained from all participants.

Data Availability Statement: We will provide the data if necessary.

Conflicts of Interest: The authors declare no conflict of interest.

\section{References}

1. Kawasaki, R.; Yasuda, M.; Song, S.J.; Chen, S.J.; Jonas, J.B.; Wang, J.J.; Mitchell, P.; Wong, T.Y. The prevalence of age-related macular degeneration in Asians: A systematic review and meta-analysis. Ophthalmology 2010, 117, 921-927. [CrossRef] [PubMed]

2. Shijo, T.; Sakurada, Y.; Fukuda, Y.; Yoneyama, S.; Sugiyama, A.; Matsubara, M.; Kikushima, W.; Tanabe, N.; Parikh, R.; Kashiwagi, K. Association of CRP levels with ARMS2 and CFH variants in age-related macular degeneration. Int. Ophthalmol. 2020, 40, 2735-2742. [CrossRef] [PubMed]

3. Brown, D.M.; Kaiser, P.K.; Michels, M.; Soubrane, G.; Heier, J.S.; Kim, R.Y.; Sy, J.P.; Schneider, S. Ranibizumab versus verteporfin for neovascular age-related macular degeneration. N. Engl. J. Med. 2006, 355, 1432-1444. [CrossRef] [PubMed]

4. Rosenfeld, P.J.; Brown, D.M.; Heier, J.S.; Boyer, D.S.; Kaiser, P.K.; Chung, C.Y.; Kim, R.Y. Ranibizumab for neovascular age-related macular degeneration. N. Engl. J. Med. 2006, 355, 1419-1431. [CrossRef] [PubMed]

5. Regillo, C.D.; Brown, D.M.; Abraham, P.; Yue, H.; Ianchulev, T.; Schneider, S.; Shams, N.; PIER Study Group. Randomized, double-masked, sham-controlled trial of ranibizumab for neovascular age-related macular degeneration: PIER Study year 1. Am. J. Ophthalmol. 2008, 145, 239-248. [CrossRef] [PubMed]

6. Lalwani, G.A.; Rosenfeld, P.J.; Fung, A.E.; Dubovy, S.R.; Michels, S.; Feuer, W.; Davis, J.L.; Flynn, H.W., Jr.; Esquiabro, M. A variable-dosing regimen with intravitreal ranibizumab for neovascular age-related macular degeneration: Year 2 of the PrONTO Study. Am. J. Ophthalmol. 2009, 148, 43-58.e1. [CrossRef] [PubMed]

7. CATT Research Group. Ranibizumab and bevacizumab for neovascular age-related macular degeneration. N. Engl. J. Med. 2011, 364, 1897-1908. [CrossRef] [PubMed]

8. Heier, J.S.; Brown, D.M.; Chong, V.; Korobelnik, J.F.; Kaiser, P.K.; Nguyen, Q.D.; Kirchhof, B.; Ho, A.; Ogura, Y.; Yancopoulos, G.D.; et al. Intravitreal aflibercept (VEGF trap-eye) in wet age-related macular degeneration. Ophthalmology 2012, 119, $2537-2548$. [CrossRef] [PubMed]

9. Minami, S.; Nagai, N.; Suzuki, M.; Kurihara, T.; Sonobe, H.; Kamoshita, M.; Uchida, A.; Shinoda, H.; Takagi, H.; Sonoda, S.; et al. Benefits of aflibercept treatment for age-related macular degeneration patients with good best-corrected visual acuity at baseline. Sci. Rep. 2018, 8, 58. [CrossRef] [PubMed]

10. Abedi, F.; Wickremasinghe, S.; Islam, A.F.; Inglis, K.M.; Guymer, R.H. Anti-VEGF treatment in neovascular age-related macular degeneration: A treat-and-extend protocol over 2 years. Retina 2014, 34, 1531-1538. [CrossRef] [PubMed]

11. Kikushima, W.; Sakurada, Y.; Sugiyama, A.; Tanabe, N.; Kume, A.; Iijima, H. Factors Predictive of Visual Outcome 1 Year after Intravitreal Aflibercept Injection for Typical Neovascular Age-Related Macular Degeneration. J. Ocul. Pharm. Ther. 2016, 32, 376-382. [CrossRef] [PubMed]

12. Yoneyama, S.; Sakurada, Y.; Kikushima, W.; Sugiyama, A.; Matsubara, M.; Fukuda, Y.; Tanabe, N.; Parikh, R.; Mabuchi, F.; Kashiwagi, K.; et al. Genetic factors associated with response to as-needed aflibercept therapy for typical neovascular age-related macular degeneration and polypoidal choroidal vasculopathy. Sci. Rep. 2020, 10, 7188. [CrossRef] [PubMed]

13. Shijo, T.; Sakurada, Y.; Yoneyama, S.; Kikushima, W.; Sugiyama, A.; Matsubara, M.; Fukuda, Y.; Mabuchi, F.; Kashiwagi, K. Association between Polygenic Risk Score and One-Year Outcomes Following As-Needed Aflibercept Therapy for Exudative Age-Related Macular Degeneration. Pharmaceuticals 2020, 13, 257. [CrossRef] [PubMed]

14. Khanani, A.M.; Gahn, G.M.; Koci, M.M.; Dang, J.M.; Brown, S.M.; Hill, L.F. Five-year outcomes of intravitreal drug therapy for neovascular age-related macular degeneration in eyes with baseline vision 20/60 or better. Clin. Ophthalmol. 2019, 13, 347-351. [CrossRef] [PubMed]

15. Javidi, S.; Dirani, A.; Antaki, F.; Saab, M.; Rahali, S.; Cordahi, G. Long-Term Visual Outcomes for a Treat-and-Extend Antivascular Endothelial Growth Factor Regimen in Eyes with Neovascular Age-Related Macular Degeneration: Up to Seven-Year Follow-Up. J. Ophthalmol. 2020, 2020, 3207614. [CrossRef] [PubMed] 
16. Maguire, M.G.; Martin, D.F.; Ying, G.S.; Jaffe, G.J.; Daniel, E.; Grunwald, J.E.; Toth, C.A.; Ferris, F.L., III; Fine, S.L.; Comparison of Age-related Macular Degeneration Treatments Trials (CATT) Research Group. Five-Year Outcomes with Anti-Vascular Endothelial Growth Factor Treatment of Neovascular Age-Related Macular Degeneration: The Comparison of Age-Related Macular Degeneration Treatments Trials. Ophthalmology 2016, 123, 1751-1761. [CrossRef] [PubMed]

17. Kikushima, W.; Sakurada, Y.; Yoneyama, S.; Sugiyama, A.; Tanabe, N.; Kume, A.; Mabuchi, F.; Iijima, H. Incidence and risk factors of retreatment after three-monthly aflibercept therapy for exudative age-related macular degeneration. Sci. Rep. 2017, 7, 44020. [CrossRef] [PubMed]

18. Kikushima, W.; Sakurada, Y.; Sugiyama, A.; Yoneyama, S.; Tanabe, N.; Matsubara, M.; Mabuchi, F.; Iijima, H. Comparison of two-year outcomes after photodynamic therapy with ranibizumab or aflibercept for polypoidal choroidal vasculopathy. Sci. Rep. 2017, 7, 16461. [CrossRef] [PubMed]

19. Wataru, K.; Sugiyama, A.; Yoneyama, S.; Matsubara, M.; Fukuda, Y.; Parikh, R.; Sakurada, Y. Five-year outcomes of photodynamic therapy combined with intravitreal injection of ranibizumab or aflibercept for polypoidal choroidal vasculopathy. PLoS ONE 2020, 15, e0229231. [CrossRef] [PubMed] 